\title{
Significance and impact of dietary factors on systemic lupus erythematosus pathogenesis (Review)
}

\author{
MARIA-MAGDALENA CONSTANTIN ${ }^{1,2}$, IULIANA ELENA NITA ${ }^{2}$, RODICA OLTEANU ${ }^{2}$, \\ TRAIAN CONSTANTIN ${ }^{1}$, STEFANA BUCUR ${ }^{2}$, CLARA MATEI $^{1}$ and ANCA RADUCAN ${ }^{3}$ \\ ${ }^{1}$ University of Medicine and Pharmacy 'Carol Davila', 050474 Bucharest; \\ ${ }^{2}$ The Second Department of Dermatology, Colentina Clinical Hospital, 020125 Bucharest; \\ ${ }^{3}$ Dr. Anca Răducan Anti-Aging Dermatology Clinic, 900162 Constanta, Romania
}

Received July 3, 2018; Accepted August 14, 2018

DOI: $10.3892 / \mathrm{etm} .2018 .6986$

\begin{abstract}
Systemic lupus erythematosus (SLE) is an autoimmune disease of unknown etiology, although its mechanisms involve genetic, epigenetic and environmental risk factors. Considering that SLE pathogenesis is yet to be explored, recent studies aimed to investigate the impact of diet, in terms of triggering or altering the course of the disease. To study the impact of diet on SLE pathogenesis, we conducted a search on Pubmed using the keywords 'diet and autoimmune diseases', 'diet and lupus', 'caloric restriction and lupus', 'polyunsaturated fatty acids and lupus', 'vitamin D and lupus', 'vitamin C and lupus' 'vitamin $\mathrm{E}$ and lupus' 'vitamin $\mathrm{A}$ and lupus' 'vitamin B and lupus', 'polyphenols and lupus', 'isoflavones and lupus', 'minerals and lupus', 'aminoacids and lupus', 'curcumin and lupus' and found 10,215 papers, from which we selected 47 relevant articles. The paper clearly emphasizes the beneficial role of personalized diet in patients with SLE, and the information presented could be used in daily practice. Proper diet in SLE can help preserve the body's homeostasis, increase the period of remission, prevent adverse effects of medication and improve the patient's physical and mental well-being.
\end{abstract}

\section{Contents}

1. Introduction

2. Search methods

3. Dietary factors

4. Discussion

Correspondence to: Dr Iuliana Elena Nita, The Second Department of Dermatology, Colentina Clinical Hospital, 19-21 Stefan cel Mare Street, 020125 Bucharest, Romania

E-mail: nitaiulianaelena@yahoo.com

Key words: autoimmune diseases, aminoacids, caloric restriction, diet, minerals, systemic lupus erythematosus

\section{Introduction}

Systemic lupus erythematosus (SLE) is the prototype of autoimmune disease, of unknown etiology, although its mechanisms involve genetic, epigenetic and environmental risk factors. Considering that SLE pathogenesis is yet to be explored, recent studies aimed to investigate the impact of diet on the disease, in terms of triggering or altering the course of SLE $(1,2)$.

There are trillions of germs inside the human gut, the microbiota, on which scientists' attention has been focused on, due to its potential to elicit the development of autoimmune disease in genetically predisposed individuals, such as inflammatory bowel disease, rheumatoid arthritis, multiple sclerosis, type 1 diabetes $(2,3)$. However, the pathogenic role of the microbiota in SLE is yet to be revealed $(4,5)$.

\section{Search methods}

To study the impact of diet on SLE pathogenesis, we conducted a search on Pubmed on October 2017 using keywords 'diet and autoimmune diseases', 'diet and lupus', 'caloric restriction and lupus', 'polyunsaturated fatty acids and lupus', 'vitamin D and lupus', 'vitamin C and lupus', 'vitamin E and lupus', 'vitamin A and lupus', 'vitamin B and lupus', 'polyphenols and lupus', 'isoflavones and lupus', 'minerals and lupus', 'aminoacids and lupus' respectively 'curcumin and lupus' and identified 7,071, 403, 13, 441, 519, 46, 53, 62, 216, 6, 4, 143, 1,227 , respectively, 11 articles matching search criteria. From a total of 10,215 papers, we reviewed the most relevant and updated articles and eliminated the papers we considered to be irrelevant for the design of our study, based on the title and abstract. We took into account publisher's prestige, credibility and prestige of the journal and validity and importance of data presented. In total, 9,521 records were excluded after reading the title and year of publication, 474 records were excluded after abstract reading, 92 full-text articles were excluded, 128 records were screened and 55 records were included. All publications included were in English.

Given that this review was limited to studies published in the English language and indexed in only one database, PubMed, we may have missed some important articles. 


\section{Dietary factors}

Research on dietary influences on SLE has focused on vitamin D, vitamin A and polyunsaturated fatty acids. Dietary changes in SLE, meaning diet supplementation with vitamins A, D, E, polyunsaturated fatty acids and phytoestrogens showed a decrease in proteinuria and glomerulonephritis in animal models (5).

There is now clear evidence that environmental factors have a high influence on SLE development, since there is a lower prevalence of the disease in West Africans than in African Americans, though both groups have the same ethnicity. The proposed theories to support this difference include the use of antibiotics and the hygiene hypothesis, which lead to the removal of some species of microbes that may have a protective role against SLE (2).

The outstanding role that food plays is sustained not only by its nutritional value, but also by its capacity to modify the structure and function of the gut microbiota.

An adequate diet is also important to help fight the associated comorbidities in SLE which increase the cardiovascular risk: diabetes mellitus, metabolic syndrome, dyslipidemia and obesity (6).

The lipid profile alterations due to medication (chronic corticotherapy) or as a result of disease activity, are aggravated by hyperlipid diet (7). Higher levels of total cholesterol and very-low-density lipoprotein cholesterol (VLDL-C), an increase in triglycerides (TG) and a decrease in high-density lipoprotein cholesterol (HDL-C) have been noted (8).

Caloric restriction. At the moment, information regarding the impact of diet on autoimmune diseases is yet insufficient. However, caloric restriction was proven to induce various benefits to the immune system, since this restriction also leads to changes in the gut microbiota (5).

Obesity among SLE adults is a real concern, since up to $40-50 \%$ of these patients are obese and more prone to suffer from fatigability (9-11). For this group of SLE patients, losing weight by keeping a low glycemic index or a low-calorie diet also proved to be efficient in lowering the level of fatigue, though the disease activity was not influenced by diet (12).

Reducing the caloric intake has helped to prevent the disease progression and the antiphospholipid syndrome in mice. In humans, one study monitoring the dietary impact of two isocaloric diets on SLE emphasized that the diet with higher level of polyunsaturated fatty acids (PUFA) reduce the risk of fetal loss and symptoms in women with SLE and antiphospholipid syndrome (13).

Strong evidence supports the idea that hypocaloric diet is positively related to disease activity. Therefore, the adequate caloric ratio for women is around 1,000-1,200 kcal/day and for men 1,200-1,400 kcal/day, with special regards to obese patients or with a tendency towards obesity, as well as SLE patients undergoing long-term or high-dose corticotherapy (14).

Other lifestyle habits that may help alleviate symptoms in SLE enclose smoking cessation and physical exercise, preferably aerobic, since it is safer (15). It may also be useful for patients with SLE to benefit from individualized nutrition counseling, which was shown to be effective in initiating dietary changes (16).
Polyunsaturated fatty acids. In patients with stable disease a diet rich in polyunsaturated fatty acids may have a positive impact on overall clinical status (15). Omega-3 fatty acids, such as eicosapentanoic acid and docosahexanoic acid, elicit an anti-inflammatory effect by decreasing the level of $\mathrm{C}$ reactive protein (CRP) and other inflammatory mediators $(17,18)$. Caloric restriction and well-established diet supplementation with omega-3 PUFA, eicosapentanoic acid and docosahexanoic acid (at a ratio of 3:1), regulates levels of total cholesterol, LDL-C and TG. Apart from the anti-inflammatory effect, adding omega-3 PUFA in SLE patients diet protects against free radicals and helps defend cardiovascular alteration by reducing the level of antibodies (anti-dsDNA), interleukins (IL-1 $\alpha$, IL-1 $\beta$, IL-2) and TNF- $\alpha$, and regulating proteinuria and blood pressure $(6,18)$. Other omega-3 acids like $\alpha$-linolenic acid and Y-linolenic acid also have beneficial effects by limiting TNF- $\alpha$ and IL-2 secretion. No downsides of introducing omega-3 PUFA to SLE patients' diet were found, as opposed to omega-6 PUFA supplementation, which may exacerbate SLE activity (6).

The main sources for omega-3 PUFA are krill oil, fish oil, olive oil, canola oil, flaxseed oil, fish (salmon, tuna, sardine, herring), but it may be found in primrose oil and soybean oil $(6,19)$. Krill oil extracted from the Antarctic krill is considered superior to fish oil since it contains a higher amount of omega-3 PUFA and it has an antioxidant effect, reducing the inflammatory joints infiltrate (19).

With a concentration of $70 \%$ omega-3 PUFA and rich in $\alpha$-linolenic acid, flaxseed oil daily intake of $30 \mathrm{~g}$ can lower serum creatinine in SLE patients with renal dysfunction $(6,20)$. High dosage of fish oil (18g/day) is also beneficial in reducing TG level to up to $\sim 40 \%$, while increasing HDL-C by a third $(21,22)$.

Omega-3 PUFAs inhibit leukocyte chemotaxis, adhesion molecule expression and production of inflammatory cytokines, through the action of specialized pro-resolving mediators, such as lipoxins, resolvins, protectins, and maresins, which reduce inflammation (23). Eicosapentaenoic acid and docosahexaenoic acid, omega-3 fatty acids found in oily fish, are able to partly inhibit inflammation, but further clinical trials are required to see their effects on SLE. Dose-dependent actions of marine n-3 PUFA on inflammatory responses have not been well described, but it appears that at least $2 \mathrm{~g} /$ day is necessary to achieve an anti-inflammatory effect (24).

Proteins. Moderate protein intake of $0,6 \mathrm{~g} / \mathrm{kg} /$ day is useful for improving renal function in SLE patients (25) and mouse studies led to the conclusion that a limitation in consumption of phenylalanine and tyrosine could also be of some benefit $(25,26)$. The major sources of proteins are meat and eggs.

Fibers. The daily intake of fibers found in whole cereals, fruits and vegetables, should be around $38 \mathrm{~g}$ for men and $25 \mathrm{~g}$ for women, in order to reduce post-prandial glycemia and lipids level, regulate hyperlipidemia, and lower blood pressure and $\mathrm{C}$ reactive protein. As far as fiber consumption is concerned, adequate water intake should be considered, and exceeding fiber intake has to be avoided because it can lead to low absorption of nutrients (6). 
Vitamins. Vitamin D is a steroid hormone with essential role on mineral metabolism and immune system homeostasis, and its deficiency has been associated with a higher susceptibility of SLE and more severe disease activity, especially in patients with dark skin (2,3).

Medical data show that the active form of vitamin D (calcitriol) may boost the innate immune response, regulating the $\mathrm{T}$ and $\mathrm{B}$ cell responses. Therefore, a lower level of vitamin $\mathrm{D}$ can be a risk factor for triggering not only SLE, but also other autoimmune diseases $(27,28)$.

Sunlight exposure is known to stimulate vitamin D production, but this elicits a controversy since SLE patients have photosensitivity. Patients with SLE are advised to use sunscreens daily even if they do not see the sun, $~ 80 \%$ of UV penetrates the clouds and the fog (29). In this case, dietary supplementation of vitamin D may be beneficial (30). In fact, some studies postulate that high doses of vitamin D (up to $50,000 \mathrm{IU} /$ day) have a preventive role, but similar improvements were obtained with doses of 2,000 IU/day $(2,31)$.

According to Antico et al (28) several studies revealed that the level of vitamin D in SLE patients was $<30 \mathrm{ng} / \mathrm{ml}$ in $56-75 \%$ of cases, whereas the same low level was found at a lower rate in healthy individuals (36-55\%). This may be due to the fact that SLE patients avoid sun exposure. Ritterhouse et al (27) stated that healthy individuals with positive ANA have a higher predisposition to vitamin D deficiency than ANA negative persons.

Low levels of vitamin D are associated to high score of SLE activity-SLEDAI (32). Vitamin D supplementation brings significant beneficial effects on bone mineral density if it can reach a plasma level of $>36,8 \mathrm{ng} / \mathrm{l}$ (30) but also on dendritic cells maturation and activation (33).

Several studies conducted on vitamin D supplementation ( $2 \mu \mathrm{g} / \mathrm{day}$ of cholecalciferol and/or alfacalcidol) have not proven any improvement in the clinical outcome of patients, nor in reducing the frequency of lupus flares (28). There was also a study aimed to examine whether vitamin D supplementation in adolescence can influence adult onset of SLE, but no associations were found (34).

In addition, one study suggests that a high level of vitamin D can also help against fatigability in SLE patients (35). However, several other studies have shown that vitamin D supplementation does not improve fatigue in SLE patients $(36,37)$.

Vitamin E, especially combined with omega-3 PUFA from fish oil, decreases levels of inflammatory cytokines, IL-2, IL-4 and TNF- $\alpha$ (6).

Vitamin C, an important antioxidant, prevents oxidative stress, reduces inflammation and lowers antibodies levels (anti-dsDNA, IgG), also preventing cardiovascular complications. Therefore, vitamin $\mathrm{C}$ should be supplemented in SLE patients diet at a maximum dose of $1 \mathrm{~g} /$ day, or in combination with vitamin $\mathrm{E}$ (vitamin C; $500 \mathrm{mg}$ and vitamin E; $800 \mathrm{IU}$ ), due to its synergic action. Orange juice, tangerine, papaya and broccoli are excellent sources of vitamin C (6).

Retinoic acid, a vitamin A metabolite, has antineoplastic effect, inhibits Th-17 and reduces antibodies level, and therefore diet supplementation should include 100,000 IU vitamin A daily, with caution not to exceed this dosage, causing symptoms that range from dry skin, alopecia, headache, nausea and anemia, to death $(6,21)$. Natural sources of vitamin A include mainly carrots and pumpkins, but it can be found in spinach, sweet potato and liver (6).

The vitamin B complex helps reduce the level of TG and LDL-C, and improves clinical symptoms in SLE. The best food supply for vitamin B is red meat, liver and fortified cereals, but it can also be found in chicken, salmon, sardine, nuts, eggs, banana, and avocado (6).

Polyphenols, isoflavones. Little information is known about polyphenols, bioactive components of a wide range of food, such as fruits and vegetables, red wine and tea, with a highly beneficial impact on the gut microbiota (12).

A recent study was set to determine the associations between polyphenols intake and fecal microbiota in patients with SLE and in controls (38).

Flavonoids represent the largest class of polyphenols and are classified in six subclasses including flavones, flavonones, flavonols, chalcones, anthocyanins and isoflavonoids. These incredible health boosters, apart from promoting a better immune response, have a well-established antioxidant and antimicrobial effect, as well as an antiaging role (39).

The main food that provide an intake of flavones include various fruits and vegetables (oranges, lettuce, watermelon, kiwi, tomato, apple, lentils, celery). The study of Cuervo et al (38), showed oranges provide the main amount of flavonones in SLE patients, while dihydrochalcones were ensured merely from apple intake. Dihydroflavonols came predominantly from consumption of red wine, whereas flavonols intake was due to tea, apple, spinach, walnut, white bean, lettuce, asparagus, broccoli, green bean and tomato. The study points out that a well-balanced diet, with a high intake of apples and oranges, as well as other fruits and vegetables rich in flavonoids, has been associated with the fecal levels of beneficial microorganisms (Lactobacillus, Blautia and Bifidobacterium) in SLE patients (38).

Isoflavones are estrogen-like nutrients, extracted from soybean, which have an antiinflammatory and antioxidant effect, reduce proteinuria and antibodies production, and can decrease IFN-Y secretion (39). Other sources of isoflavones are black beans, olive oil and cereals (6).

Minerals. Special attention is to be paid to mineral intake since it is best to restrict consumption of some minerals such as zinc and sodium. It was emphasized that a decrease in zinc can improve symptoms in SLE patients and also reduce levels of antibodies (anti-dsDNA) (22). Zinc is particularly found in mollusks, but also in milk, soybean and spinach (6).

Sodium intake not only has no beneficial effect but also exacerbates renal dysfunction (40) in SLE patients, who should be advised to reduce salt and condiments from their diet. For these patients, sodium intake should be less than $3 \mathrm{~g} /$ day (41).

Selenium has antioxidant and anti-inflammatory effects and can be added to patients' diet from consumption of nuts, whole cereals, eggs and ricotta (6). Calcium levels can be increased by the intake of diary, spinach, sardine or soybean, but oral supplements are also of use (Calcium >1500 mg daily, in addition to vitamin D; 800 IU vitamin D) in order to help prevent bone mass loss. Iron should only be used in anemic patients, to maintain a balance, since excess of iron can 
aggravate renal impairment in SLE patients and a deficiency exacerbates clinical symptomatology (6).

Aminoacids and other nutrients. Conflicting results have emerged concerning L-canavanine, a non-proteicaminoacid that controls antibodies synthesis and has a suppressive effect on $\mathrm{T}$ cells. The main source for L-canavanine are soybean and alfalfa, but it can also be extracted from onion (6). Some studies revealed that alfalfa sprouts induced lupus-like syndrome in otherwise healthy individuals and reactivated SLE both clinically and serologically in patients with inactive disease (42).

Taurine, found in meat, eggs and oyster, is a $\beta$ aminoacid which regulates the immune response, decreases the oxidative stress and lowers the level of inflammatory cytokines and lipids (6).

Royal jelly, rich in aminoacids and vitamins, could supplement SLA patients' diet, due to its hypocholesterolemic, anti-inflammatory and immunoregulatory effects (43).

Over the past decades, curcumin, a polyphenol extracted from a spice called Curcuma longa, also known as turmeric, has been highly studied in both pre-clinical and clinical trials (44). Due to its well-established anti-inflammatory, antioxidant, antibacterial, hypoglycemic and wound healing effects $(45,46)$ curcumin has been used in a wide range of inflammatory diseases including the dermatological spectrum (psoriasis, vitiligo, SLE- lupus nephritis, oral lichen planus) (44).

The recommended dosage for lupus nephritis is $500 \mathrm{mg}$ daily for 3 months, which leads to a reduction in proteinuria, hematuria and blood pressure in SLE patients who have relapsing of intractable lupus nephritis (47). Curcumin modulates pro-inflammatory cytokines, adhesion molecules and CRP, thus eliciting a beneficial anti-inflammatory effect in arthritis, by reducing pain and CRP level, and increasing the walking distance, at a dosage of $200 \mathrm{mg}$ daily for 3 months $(48,49)$. When used in combination with celecoxib (COX-2 inhibitor), the effect is synergic (50). The dosage for SLE ranges from 100-200 mg daily to $4.5 \mathrm{~g} /$ day, but curcumin supplementation is considered safe in up to $12 \mathrm{~g}$ daily (44).

\section{Discussion}

Patients' lifestyle may adjust the course of SLE, because diet or psychological stress are involved in the modulation of cutaneous inflammation (51). By small improvements in diet, the state may lower the costs arising from hospitalization and administration of drugs to patients with SLE (52).

Taking into account the results of the ample studies that we have discussed, we support the importance of diet on systemic lupus and we propose a dietary plan. Therefore, diet for patients with lupus should be personalized. In the era of expensive biologicals or biosimilars $(53,54)$ diet could improve clinical status of patients with SLE in a cost-effective way.

Caloric restriction is beneficial to the immune system, it is positively related to disease activity and it lowers the level of fatigue. Diets with higher level of PUFA reduce the risk of fetal loss and symptoms in women with SLE and antiphospholipid syndrome, and it may also have a positive impact on overall clinical status. Omega-3 reduces cardiovascular risk, while omega-6 PUFA may exacerbate SLE activity. Flaxseed oil can lower serum creatinine and fish oil reduce TG and increase
HDL-C. Moderate protein intake improves renal function. Fibers regulate hyperlipidemia, lower blood pressure and CRP. Vitamins are also important, vitamin D deficiency is associated with more severe disease activity, vitamin $\mathrm{C}$ prevents cardiovascular complications, reduces inflammation and antibodies level, retinoic acid also reduces antibodies level and vitamins from B complex improves clinical symptoms, reduce TG and LDL-C. Flavonoids reduce proteinuria, antibodies production and INF- $\gamma$ production. Regarding minerals, it's best to restrict zinc and sodium consumption, and also excess of iron. Curcumin (turmeric) is beneficial to lupus nephritis.

Patients with SLE should introduce in their diet whole grains instead of refined ones (also adding barley, quinoa and rye), sea salt instead of refined salt, and instead of sugar, patients should use rice, barley, or maple syrup. Fresh vegetables should be consumed daily, as well as at least one fruit per day. Fresh fish also play a beneficial role in SLE patient's diet and should be added to the personalized diet, as well as cold pressed oil. Patients can supplement the diet with flaxseeds, pumpkins, carrots, nuts, oranges or apples.

In conclusion, the paper clearly emphasizes the beneficial role of personalized diet in patients with SLE, and the information presented could be successfully used in our daily practice, helping patients understand the dietary impact on their disease and establishing individualized diet plans.

Proper diet in SLE can help preserve the body's homeostasis, increase the period of remission, prevent adverse effects of medication (especially systemic corticotherapy), and improve the patient's physical and mental well-being.

However, further prospective studies are needed on large cohorts of patients to quantify the long-term impact of diet on SLE, and we believe this study is useful starting point in this direction.

\section{Acknowledgements}

Not applicable.

\section{Funding}

No funding was received.

\section{Availability of data and materials}

Not applicable.

\section{Authors' contributions}

MMC contributed to design of study and revision the manuscript for important intellectual content. IEN and AR were responsible for the analysis and interpretation of the data and contributed to drafting the manuscript. RO, TC and CM acquired the data and revised critically the work. SB contributed to the conception of work, revision the language and contributed to drafting the manuscript. All authors agreed to be accountable for all aspects of the work in ensuring that questions related to the accuracy or integrity of any part of the work are appropriately investigated and resolved. All authors read and approved the final manuscript. 


\section{Ethics approval and consent to participate}

Not applicable.

\section{Patient consent for publication}

Not applicable.

\section{Competing interests}

The authors declare that they have no competing interests.

\section{References}

1. Kamen DL: Environmental influences on systemic lupus erythematosus expression. Rheum Dis Clin North Am 40: 401-412, 2014.

2. Mu Q, Zhang $\mathrm{H}$ and Luo XM: SLE: Another Autoimmune Disorder Influenced by Microbes and Diet? Front Immunol 6 : $608,2015$.

3. Kamada N, Seo SU, Chen GY and Núñez G: Role of the gut microbiota in immunity and inflammatory disease. Nat Rev Immunol 13: 321-335, 2013

4. Hevia A, Milani C, López P, Cuervo A, Arboleya S, Duranti S, Turroni F, González S, Suárez A, Gueimonde M, et al: Intestinal dysbiosis associated with systemic lupus erythematosus. MBio 5 : e01548-e14, 2014. doi: 10.1128/mBio.01548-14

5. Vieira SM, Pagovich OE and Kriegel MA: Diet, microbiota and autoimmune diseases. Lupus 23: 518-526, 2014.

6. Klack K, Bonfa E and Borba Neto EF: Diet and nutritional aspects in systemic lupus erythematosus. Rev Bras Reumatol 52: 384-408, 2012

7. Caetano MC, Ortiz TT, Terreri MT, Sarni RO, Silva SG, Souza FI and Hilário MO: Inadequate dietary intake of children and adolescents with juvenile idiopathic arthritis and systemic lupus erythematosus. J Pediatr (Rio J) 85: 509-515, 2009.

8. Bruce IN: 'Not only...but also': Factors that contribute to accelerated atherosclerosis and premature coronary heart disease in systemic lupus erythematosus. Rheumatology (Oxford) 44: 1492-1502, 2005.

9. Oeser A, Chung CP, Asanuma Y, Avalos I and Stein CM: Obesity is an independent contributor to functional capacity and inflammation in systemic lupus erythematosus. Arthritis Rheum 52: 3651-3659, 2005.

10. Katz P, Gregorich S, Yazdany J, Trupin L, Julian L, Yelin E and Criswell LA: Obesity and its measurement in a community-based sample of women with systemic lupus erythematosus. Arthritis Care Res (Hoboken) 63: 261-268, 2011.

11. Katz P, Yazdany J, Julian L, Trupin L, Margaretten M, Yelin E and Criswell LA: Impact of obesity on functioning among women with systemic lupus erythematosus. Arthritis Care Res (Hoboken) 63: 1357-1364, 2011.

12. Yuen HK and Cunningham MA: Optimal management of fatigue in patients with systemic lupus erythematosus: A systematic review. Ther Clin Risk Manag 10: 775-786, 2014.

13. Reifen R, Amital H, Blank M, Sklan D, Berkovich Z, Gershwin E and Shoenfeld Y: Linseed oil suppresses the anti-beta-2-glycoprotein-I in experimental antiphospholipid syndrome. J Autoimmun 15: 381-385, 2000

14. National Institutes of Health: National Heart, Lung, and Blood Institute: Obesity education initiative. Clinical guidelines on the identification, evaluation, and treatment of overweight and obesity in adults: The evidence report. Obes Res 6: 51S-209S 1998.

15. Rodríguez Huerta MD, Trujillo-Martín MM, Rúa-Figueroa Í, Cuellar-Pompa L, Quirós-López R and Serrano-Aguilar P Spanish SLE CPG Development Group: Healthy lifestyle habits for patients with systemic lupus erythematosus: A systemic review. Semin Arthritis Rheum 45: 463-470, 2016

16. Everett ST, Wolf R, Contento I, Haiduc V, Richey M and Erkan D: Short-term patient-centered nutrition counseling impacts weight and nutrient intake in patients with systemic lupus erythematosus. Lupus 24: 1321-1326, 2015

17. Borges MC, Santos FM, Telles RW, Correia MI and Lanna CC: Polyunsaturated omega-3 fatty acids and systemic lupus erythematosus: What do we know? Rev Bras Reumatol 54: 459-466, 2014 (In Portuguese).
18. Halade GV, Rahman MM, Bhattacharya A, Barnes JL, Chandrasekar B and Fernandes G: Docosahexaenoic acid-enriched fish oil attenuates kidney disease and prolongs median and maximal life span of autoimmune lupus-prone mice. J Immunol 184: 5280-5286, 2010

19. Ierna M, Kerr A, Scales H, Berge $K$ and Griinari $M$ : Supplementation of diet with krill oil protects against experimental rheumatoid arthritis. BMC Musculoskelet Disord 11: 136 , 2010.

20. Petri M: Diet and systemic lupus erythematosus: From mouse and monkey to woman? Lupus 10: 775-777, 2001

21. Patavino T and Brady DM: Natural medicine and nutritional therapy as an alternative treatment in systemic lupus erythematosus. Altern Med Rev 6: 460-471, 2001.

22. Leiba A, Amital H, Gershwin ME and Shoenfeld Y: Diet and lupus. Lupus 10: 246-248, 2001.

23. Molfino A, Amabile MI, Monti M and Muscaritoli M: Omega-3 polyunsaturated fatty acids in critical illness: Anti-inflammatory, proresolving, or both? Oxid Med Cell Longev 2017: 5987082, 2017.

24. Calder PC: Marine omega-3 fatty acids and inflammatory processes: Effects, mechanisms and clinical relevance. Biochim Biophys Acta 1851: 469-484, 2015.

25. MilovanovIu S: Lysenko LV, Milovanova Ll, Dobrosmyslov IA: The role of balanced low-protein diet in inhibition of predialysis chronic kidney disease progression in patients with systemic diseases. Ter Arkh 81: 52-57, 2009.

26. Brown AC: Lupus erythematosus and nutrition: A review of the literature. J Ren Nutr 10: 170-183, 2000.

27. Ritterhouse LL, Lu R, Shah HB, Robertson JM, Fife DA, Maecker HT, Du H, Fathman CG, Chakravarty EF, Scofield RH, et al: Vitamin D deficiency in a multiethnic healthy control cohort and altered immune response in vitamin D deficient European-American healthy controls. PLoS One 9: e94500, 2014.

28. Antico A, Tampoia M, Tozzoli R and Bizzaro N: Can supplementation with vitamin $\mathrm{D}$ reduce the risk or modify the course of autoimmune diseases? A systematic review of the literature. Autoimmun Rev 12: 127-136, 2012.

29. Solovastru LG, Vata D, Stătescu L, Constantin MM and Andrese E: Skin cancer between myth and reality, yet ethically constrained. Rev Rom Bioet 12: 47-52, 2014.

30. Kamen DL, Cooper GS, Bouali H, Shaftman SR, Hollis BW and Gilkeson GS: Vitamin D deficiency in systemic lupus erythematosus. Autoimmun Rev 5: 114-117, 2006.

31. Arnson Y, Amital H and Shoenfeld Y: Vitamin D and autoimmunity: New aetiological and therapeutic considerations. Ann Rheum Dis 66: 1137-1142, 2007.

32. Wu PW, Rhew EY, Dyer AR, Dunlop DD, Langman CB, Price H, Sutton-Tyrrell K, McPherson DD, Edmundowicz D, Kondos GT, et al: 25 -hydroxyvitamin $\mathrm{D}$ and cardiovascular risk factors in women with systemic lupus erythematosus. Arthritis Rheum 61: $1387-1395,2009$

33. Ben-Zvi I, Aranow C, Mackay M, Stanevsky A, Kamen DL, Marinescu LM, Collins CE, Gilkeson GS, Diamond B and Hardin JA: The impact of vitamin D on dendritic cell function in patients with systemic lupus erythematosus. PLoS One 5: e9193, 2010.

34. Hiraki LT, Munger KL, Costenbader KH and Karlson EW: Dietary intake of vitamin D during adolescence and risk of adult-onset systemic lupus erythematosus and rheumatoid arthritis. Arthritis Care Res (Hoboken) 64: 1829-1836, 2012.

35. Ruiz-Irastorza G, Gordo S, Olivares N, Egurbide MV and Aguirre C: Changes in vitamin D levels in patients with systemic lupus erythematosus: Effects on fatigue, disease activity, and damage. Arthritis Care Res (Hoboken) 62: 1160-1165, 2010

36. Fragoso TS, Dantas AT, Marques CD, Rocha Junior LF, Melo JH, Costa AJ and Duarte AL: 25-Hydroxyivitamin D3 levels in patients with systemic lupus erythematosus and its association with clinical parameters and laboratory tests. Rev Bras Reumatol 52: 60-65, 2012.

37. Stockton KA, Kandiah DA, Paratz JD and Bennell KL: Fatigue, muscle strength and vitamin D status in women with systemic lupus erythematosus compared with healthy controls. Lupus 21: 271-278, 2012.

38. Cuervo A, Hevia A, López P, Suárez A, Sánchez B, Margolles A and González S: Association of polyphenols from oranges and apples with specific intestinal microorganisms in systemic lupus erythematosus patients. Nutrients 7: 1301-1317, 2015.

39. Terahara N: Flavonoids in foods: A review. Nat Prod Commun 10: 521-528, 2015. 
40. Tantu M, Belu E, Bobescu E, Armean SM, Armean P and Constantin MM: Domnariu CD6: Role of angiotensin converting enzyme (ACE) inhibitors in hypertension and cardiovascular protection management. Farmacia 62: 443-451, 2014.

41. Hull RP and Goldsmith DJ: Nephrotic syndrome in adults. BMJ 336: 1185-1189, 2008

42. Hong YH, Huang CJ, Wang SC and Lin BF: The ethyl acetate extract of alfalfa sprout ameliorates disease severity of autoimmune-prone MRL-lpr/lpr mice. Lupus 18: 206-215, 2009.

43. Mannoor MK, Shimabukuro I, Tsukamotoa M, Watanabe $H$, Yamaguchi K and Sato Y: Honeybee royal jelly inhibits autoimmunity in SLE-prone NZB x NZW F1 mice. Lupus 18: 44-52, 2009.

44. Gupta SC, Patchva S and Aggarwal BB: Therapeutic roles of curcumin: Lessons learned from clinical trials. AAPS J 15: $195-218,2013$

45. Schraufstatter E and Bernt H: Antibacterial action of curcumin and related compounds. Nature 164: 456-457, 1949.

46. Aggarwal BB and Sung B: Pharmacological basis for the role of curcumin in chronic diseases: An age-old spice with modern targets. Trends Pharmacol Sci 30: 85-94, 2009.

47. Khajehdehi P, Zanjaninejad B, Aflaki E, Nazarinia M, Azad F, Malekmakan L and Dehghanzadeh GR: Oral supplementation of turmeric decreases proteinuria, hematuria, and systolic blood pressure in patients suffering from relapsing or refractory lupus nephritis: A randomized and placebo-controlled study. J Ren Nutr 22: 50-57, 2012.
48. Belcaro G, Cesarone MR, Dugall M, Pellegrini L, Ledda A, Grossi MG, Togni S and Appendino G: Product-evaluation registry of Meriva ${ }^{\circledR}$, a curcumin-phosphatidylcholine complex, for the complementary management of osteoarthritis. Panminerva Med 52 (Suppl 1): 55-62, 2010.

49. Aggarwal BB, Gupta SC and Sung B: Curcumin: An orally bioavailable blocker of TNF and other pro-inflammatory biomarkers. Br J Pharmacol 169: 1672-1692, 2013.

50. Shpitz B, Giladi N, Sagiv E, Lev-Ari S, Liberman E, Kazanov D and Arber N: Celecoxib and curcumin additively inhibit the growth of colorectal cancer in a rat model. Digestion 74: 140-144, 2006.

51. Căruntu C, Boda D, Musat S, Căruntu A and Mandache E: Stress-induced mast cell activation in glabrous and hairy skin. Mediators Inflamm 2014: 105950, 2014.

52. Raţiu MP, Purcărea I, Popa F, Purcărea VL, Purcărea TV, Lupuleasa D and Boda D: Escaping the economic turn down through performing employees, creative leaders and growth driver capabilities in the Romanian pharmaceutical industry. Farmacia 59: 119-13, 2011.

53. Olteanu R, Zota A and Constantin M: Biosimilars: An Update on Clinical Trials (Review of Published and Ongoing Studies). Acta Dermatovenerol Croat 25: 57-66, 2017.

54. Olteanu R, Constantin MM and Zota A: Original clinical experience and approach to treatment study with interleukine $12 / 23$ inhibitor in moderate-to-severe psoriasis patients. Farmacia 64: 918-921, 2016. 